\title{
Treatment outcome and metacognitive change in CBT and GET for Chronic Fatigue Syndrome
}

Word count: 4,865 (excluding Abstract, Figures and Tables)

Bruce A. Fernie

King's College London, Institute of Psychiatry, Department of Psychology, London, UK

CASCAID, South London \&Maudsley NHS Foundation Trust, London, UK

Gabrielle Murphy

Fatigue Service, Royal Free Hampstead NHS Foundation Trust, London, UK

\author{
Adrian Wells \\ University of Manchester, Manchester, UK \\ Ana V. Nikčević \\ Kingston University, Kingston upon Thames, UK \\ Marcantonio M. Spada \\ London South Bank University, London, UK
}

Revision 6

March 2015

\section{Acknowledgements}

Author BF receives salary support from the National Institute for Health Research (NIHR) Mental Health Biomedical Research Centre and Dementia Research Unit at South London and Maudsley NHS Foundation Trust and King's College London. The views expressed are those of the author and not necessarily those of the NHS, the NIHR or the Department of Health.

\section{Thanks}

The authors wish to thank the therapists and administration staff (Andy Ajiz, Jane Atkinson, Colette Bennett, Jen Bobrow, Michael Butters, Stacy Millett-Clay, Jaclyn Cook, Ruth Cowlishaw, Lorraine Maher-Edwards, Shari Frohlich, Christina Hanson, Jason Robinson, Joanna Smee, and Judith Smit) at the Fatigue Service, both past and present, for facilitating the provision of therapy and supporting the authors in the write up of this service evaluation.

\section{Author Notes}

Correspondence should be addressed to: Marcantonio M. Spada, School of Applied Sciences, London South Bank University, United Kingdom. Tel. +44 (0)20 7815 5760, e-mail spadam@lsbu.ac.uk. 


\section{Abstract}

\section{Background}

Studies have reported that Cognitive Behavioural Therapy (CBT) and Graded Exercise Therapy (GET) are effective treatments for Chronic Fatigue Syndrome (CFS).

\section{Method}

One hundred and seventy one patients undertook a course of either CBT $(n=116)$ or GET $(n=55)$ and where assessed on a variety of self-report measures at pre- and post-treatment and follow-up.

\section{Aim}

In this paper we present analyses on treatment outcomes for CBT and GET in routine clinical practice and evaluate whether changes on subscales of the Metacognitions Questionnaire-30 (MCQ30) predict fatigue severity independently of changes in other covariates, and across the two treatment modalities.

\section{Results}

Both CBT and GET were equally effective at decreasing fatigue, anxiety, and depression, and at increasing physical functioning. Changes on the subscales of the MCQ-30 were also found to have a significant effect on fatigue severity independently of changes in other covariates and across treatment modalities.

\section{Conclusion}

The findings from the current study suggest that CFS treatment protocols for CBT and GET, based on those from the PACE trial, achieve similar-to-poorer outcomes in routine clinical practice as in a RCTs

Key words: cognitive behavioural therapy; chronic fatigue syndrome; graded exercise therapy; metacognitions; metacognitive change; outcomes evaluation. 


\section{Introduction}

Chronic Fatigue Syndrome (CFS) is a complex condition involving both biological and psychological dimensions. The constellation of symptoms that result from this condition have a wide range of detrimental impacts on an individual's quality of life. No specific test, or any physical sign for the diagnosis of CFS, exists. CFS is a diagnosis of exclusion: it is made when it is has not been possible to attribute a patient's symptoms to any other known disease process. CFS is characterised by the presence of a severe and disabling fatigue, both physical and mental, for at least 6 months, that is not life-long but has a definite onset (Sharpe et al., 1991). Currently, no aetiology for CFS has been identified, thus it can be considered part of the spectrum of Medically Unexplained Symptoms. In terms of treatment, the National Institute for Health and Clinical Excellence (NICE, 2007) recommends Cognitive Behavioural Therapy (CBT) and Graded Exercise Therapy (GET) for CFS/ME (Myalgic Encephalomyelitis), as well as suggesting pacing for the severely affected.

\subsection{CBT and GET for CFS}

The CBT model of CFS attends to factors that may predispose (e.g. perfectionism), precipitate (e.g. virus, stress, and/or trauma), and perpetuate (e.g. stress, negative emotion, and allor-nothing patterns of behaviour) symptoms. This model models incorporate fear-avoidance beliefs as a maintaining factor, driving all-or-nothing patterns of behaviour, contributing emotional distress and exacerbating symptom severity. Fear-avoidance beliefs are sometimes controversial, with some patient groups believing that they are 'victim-blaming'. However, research has suggested that changes in avoidance behaviour and related beliefs are associated with good outcome in CBT (Deale, Chalder, \& Wessely, 1998).

The GET model of CFS is based on the concept of de-conditioning. After encountering an initial trigger to CFS (such as a viral infection), maladaptive coping responses (e.g. resting to get better) lead to de-conditioning. GET encourages patients to establish an 'exercise baseline' (a duration of exercise that does not significantly exacerbate symptoms) and to gradually increase first 
it's duration and then the intensity. Research suggests that the GET mechanism of change is through the modification of interpretations about bodily sensations (Moss-Morris, Sharon, Tobin, \& Baldi, 2005).

Several RCTs have found evidence to suggest both CBT and GET are effective treatments for CFS (e.g. Moss-Morris et al., 2005; Prins et al., 2001). Recently a large multi-centred RCT compared CBT, GET and Adaptive Pacing Therapy (APT) with specialist medical care (SMC) to SMC alone (White et al., 2011) and found both GET and CBT to be moderately effective treatments for CFS. Non-significant differences were found between APT and SMC alone. However, the results of a RCT may be a consequence of the context in which they occur and may differ in routine clinical practice. Indeed, outcomes in RCTs for CFS have been found to be superior to those achieved in routine clinical practice (Quarmby, Rimes, Deale, Wessely, \& Chalder, 2007).

\subsection{The Role of Metacognitions in CFS}

Research indicates that CBT and GET are beneficial, however limited efforts have been focused on examining the effects of such treatment modalities on specific cognitive processes that are hypothesized to mediate change. Such studies are helpful to identify target treatments, consider suitability, and explore mechanisms of change.

The Self-Regulatory Executive Function (S-REF) model (Wells, 2011) suggests that vulnerability to, and maintenance of, psychological problems is associated with a cognitiveattentional syndrome (CAS) characterized by perseverative thinking (e.g. rumination and worry), threat monitoring, and self-regulation strategies that fail to modify maladaptive self-knowledge. The CAS is driven by metacognitions, which are beliefs that specify the execution of ruminative/worry processing, guide attention, and serve as a resource for interpreting and controlling cognitive events such as unwanted thoughts. A wide evidence base supports the relationship between metacognitions, aspects of the CAS, and a range of negative emotional outcomes and psychological problems (for a review see Wells, 2011). 
Recent studies have provided support for CAS configurations in CFS. For example, interpretative biases for processing somatic information (Moss-Morris \& Petrie, 2003), high levels of self-reported health-related worry and preoccupation with health threats (Aggarwal, McBeth, Zakrzewska, Lunt, \& Macfarlane, 2006), together with attentional biases for processing healththreat information (Hou, Moss-Morris, Bradley, Peveler, \& Mogg, 2008), have been found to be present in CFS patients. For a review of these cognitive processes see Knoop, Prins, Moss-Morris, and Bleijenberg (2010).

Other studies have posited a role for metacognitions in CFS. Specifically, metacognitions regarding lack of cognitive confidence and need to control thoughts have been found to predict CFS symptom severity when controlling for negative emotion (Maher-Edwards, Fernie, Murphy, Wells, \& Spada, 2011). Metacognitions pertaining to the need to control thoughts may lead to the activation of effortful and maladaptive coping strategies (e.g. thought suppression, worry, and rumination) that may increase fatigue and negative appraisals of cognitive experiences. Metacognitions regarding a lack of cognitive confidence may limit choice and inhibit activation of adaptive coping strategies when feeling fatigued. Additionally, metacognitions pertaining to perserverative conceptual thinking about symptoms and body-focussed attention were elicited from individuals with CFS (Maher-Edwards, Fernie, Murphy, Nikcevic, \& Spada, 2012).

\subsection{Aims of the Current Study}

The aims of the current study were to analyse outcomes for both CBT and GET for CFS in routine clinical practice (i.e. within a specialist fatigue service) as well as to examine metacognitive change as a predictor of treatment outcome. The central hypothesis tested was that reductions in metacognitions occurring within treatment for CFS would be associated with improvements in fatigue severity independently of changes in other covariates, and across the two therapeutic modalities. 


\section{Method}

\subsection{Patients}

One hundred and seventy one patients began a course of either CBT $(n=116)$ or GET $(n=55)$ at the Fatigue Service at the Royal Free Hospital, London, UK, between the $12^{\text {th }}$ April 2010 and the $4^{\text {th }}$ March 2013. They provided signed consent for their data to be used in a service evaluation. Of the 171 patients who started therapy, 148 completed therapy (103 CBT and 45 GET patients), representing 86.5\% completion rate, attending a mean of 13.7 (CBT) and 13.4 (GET) sessions. 10 CBT patients and seven GET dropped out of therapy without agreeing to do so first with their therapist. Three patients each from CBT and GET ended therapy with the consent of their therapist. The mean age of patients was 40.8 years ( $\mathrm{SD}=12.5$; range 18-75). Patients with a diagnosis of CFS according to the Oxford Criteria (Sharpe et al., 1991) were included in this study. Table 1 shows fatigue, physical functioning, anxiety, and depression baseline scores for CBT and GET patients.

\subsection{Self-report Measures}

\section{Chalder Fatigue Questionnaire [CFQ (Chalder et al., 1993)]}

The CFQ is an 11-item self-report measure that assesses levels of mental (e.g. "Do you need to rest more?”) and physical (e.g. Do you have problems with tiredness?”) fatigue over the previous month. The measure employs a four-point Likert-type response format to which respondents are required to indicate the extent the severity of their different symptoms compared to 'usual'. Higher scores indicate higher levels of mental and physical fatigue. The CFQ can be scored in two ways: (1) using a bimodal system $(0,0,1,1)$, with a possible range of $0-11$; or (2) using system where each possible response is assigned either $0,1,2$ or 3 point value, producing a possible range of 0 33. This study utilises the four-point scoring system. The CFQ possesses good psychometric properties (Chalder et al., 1993) and is recommended for use in the Adult Minimum Dataset for assessing severity of CFS/ME (NICE, 2007). 
RAND Short Form 36 Physical Functioning [SF-36 PF (Ware Jr \& Sherbourne, 1992)]

The SF-36 PF is a 10-item self-report measure assessing daily activities such as climbing stairs, lifting or carrying groceries, walking, and bathing. It is the Physical Functioning subscale of the RAND Short Form 36 Health Questionnaire that assesses function in different areas such as general health, social functioning, mental health and limitations in role activities because of physical or emotional problems (Ware Jr \& Sherbourne, 1992). Participants indicate how much their health limits them in these activities, responding to individual items by choosing between "a lot”, “a little” or "not at all”. Higher scores indicate higher levels of physical functioning. The SF36 PF is a widely used, reliable, and valid measure with population norms and normative data for a variety of medical conditions (Ware \& Kosinski, 2001).

Hospital Anxiety and Depression Scale [HADS (Zigmond \& Snaith, 1983)]

The HADS is a 14-item self-report measure that assesses anxiety and depression. The anxiety factor (7 items) consists of items like "I get a sort of frightened feeling as if something horrible is about to happen". The depression factor (7 items) consists of items like "I feel as if I am slowed down”. Higher scores indicate higher levels of anxiety and depression. The HADS possesses good psychometric properties (Mykletun, 2001; Zigmond \& Snaith, 1983).

Metacognitions Questionnaire 30 [MCQ-30 (Wells \& Cartwright-Hatton, 2004)]

The MCQ-30 is a 30-item self-report measure that assesses five-factors pertaining to metacognition: (1) positive beliefs about worry (e.g. "Worrying helps me cope”); (2) negative beliefs about thoughts concerning uncontrollability and danger (e.g. "When I start worrying I cannot stop”); (3) cognitive confidence (e.g. "My memory can mislead me at times”); (4) beliefs about the need to control thoughts (e.g. "Not being able to control my thoughts is a sign of weakness”); and (5) cognitive self-consciousness (e.g. "I pay close attention to the way my mind works”). Respondents are required to indicate the extent of their agreement with the metacognitions presented on a four-point Likert-type answer system. Higher scores indicate higher levels of unhelpful metacognitions. The MCQ-30 possesses good psychometric properties (Spada, 
Mohiyeddini, \& Wells, 2008; Wells \& Cartwright-Hatton, 2004). Reliability analysis on MCQ-30 responses from this sample indicates that it possesses good-to-excellent internal consistency (i.e. Cronbach's alphas for each of the five factors were $.89, .91, .92, .83$, and .87 respectively). These are similar Cronbach’s alphas to those obtained by Maher-Edwards et al. (2011).

\section{Clinical Global Impression [CGI (Guy, 1976)]}

This is a single item self-report measure that assesses change in the severity of fatigue symptoms over the previous 3 months on a seven-point Likert scale ('very much better', 'much better' 'a little better', 'no change', ‘a little worse', 'much worse’ and ‘very much worse’).

\section{Therapy Satisfaction Scale [TSS]}

This is a single item self-report measure that assesses satisfaction with therapy on a sevenpoint Likert scale ('very satisfied', 'satisfied', 'a little satisfied', 'neither satisfied nor dissatisfied', 'a little dissatisfied', 'dissatisfied’ and 'very dissatisfied').

\subsection{Procedure and Care Pathway}

Patients were referred to the service via their GP and then assessed by the team physician for a CFS diagnosis. Patients interested in therapy were offered a further assessment conducted by a team therapist. Patient preference drove choice of therapy, and this decision would be confirmed during the clinical team's weekly multidisciplinary meeting. Following assessment patients were given the pre-treatment self-report measures booklet. Patients were then contacted either by telephone or mail to arrange their first session of therapy.

14 sessions of either CBT or GET were administered. The first 12 of these sessions were typically weekly or fortnightly. Following session 12 patients completed the post-treatment selfreport measures booklet. Sessions 13 or 14 were follow-up or booster sessions, typically occurring at four to eight week intervals after session 12. Patients completed their follow-up self-report measures booklet at or shortly after session 14. On treatment completion, a physician or clinical nurse specialist conducted a medical review. 
Following the submission of a brief proposal and consultation with the Research and Development team at the hospital, this study was deemed a service evaluation and therefore did not need to obtain ethics approval.

\subsection{CBT and GET Treatment Protocols}

Both CBT and GET treatment protocols followed closely the treatment protocols used in the PACE trial (White et al., 2011), outlined in (White, Sharpe, Chalder, DeCesare, \& Walwyn, 2007). CBT therapists were all qualified or trainee clinical and counselling psychologists and supervised by a senior psychologist (first author) who specializes in CBT. GET therapists were either physiologists or exercise physiologists and were supervised by a senior physiotherapist for GETrelated issues and by the first author for therapy relationship-issues. Supervision frequency was based on relevant professional guidelines as a minimum. Three therapists (three) had been involved in the PACE trial, either providing therapy or as a co-supervisor. A total of six psychologists (not working concurrently) contributed data to this evaluation, along with one physiotherapist and one exercise physiologist.

CBT and GET shared many components including the use of activity and sleep diaries, activity scheduling with graded increases in activity, promotion of sleep hygiene, and relaxation exercises. Differences centred on GET's focus on exercise baselines and CBT's attention to coping strategies and challenging unhelpful beliefs about symptoms and activity.

\subsection{Data Analysis}

The data analysis strategy used, where possible, mirrored that utilised by the PACE trial (White et al., 2011). Primary outcome variables used continuous outcomes and these measures were prorated by adding a mean score value for missing items if a maximum of two scores were missing. Difference tests were conducted on baseline primary and secondary outcome data between therapies. Four linear mixed-modelling regression analyses were conducted using Statistical Package for the Social Sciences version 20 (IBM, 2011). These had fatigue (CFQ), physical functioning (SF-36 PF), and both anxiety and depression (HADS) as outcome variables. The 
variable 'time' was created to represent the index for repeated measurements (pre-treatment, posttreatment, and follow-up) and was entered as a fixed effect in all models, as was treatment modality. Each patient was given a random slope and intercept to allow a unique growth trajectory. The first model generated, with fatigue as the outcome variable, had change in anxiety, depression, beliefs concerning uncontrollability and danger of thoughts, and cognitive confidence covariates entered as fixed effects. These covariates were chosen for the first model on the basis of a nonparametric correlation matrix that explored relationships between the change in fatigue scores and changes in all measured variables between pre-treatment and follow-up. Several models were developed that controlled for negative affect and type of therapy, with different combinations of metacognitive covariates as fixed effects. The model presented was chosen on the basis of measures of model strength, power, and ease of interpretation.

We recoded the CGI and TSS into three levels and presented these as percentages. In line with the PACE trial's presentation of this data, CGI responses of 'very much better' and 'much better' were coded as 'positive change’; ‘a little better', 'no change' and 'a little worse' were coded as 'minimal change'; and 'much worse' and 'very much worse' were coded as 'negative change'. TSS responses of 'very satisfied' and 'satisfied' were coded as 'satisfied'; 'a little satisfied', 'neither satisfied' and 'a little dissatisfied' were coded as 'mostly indifferent'; while 'dissatisfied' and 'very dissatisfied' were coded as 'dissatisfied'.

\section{Results}

Kolmogorov-Smirnov tests suggested that all primary and secondary outcomes were significantly different from normal. As result, a series of Mann-Whitney tests were conducted on the baseline values of these variables. These revealed a significant difference between baseline values of physical functioning [U=2340; $p=.009$ ] (with lower functioning in GET patients) and anxiety [U=2266, $\mathrm{p}=.002]$ (with greater anxiety on CBT patients). No significant differences were found between CBT and GET patients in fatigue [U=3033, $\mathrm{p}>.05$ ] and depression [U=2844, $\mathrm{p}>.05$ ]. 
Table 1 shows the mean scores of the primary (CFQ and SF-36 PF) and secondary (HADS) outcome measures, suggesting that levels of fatigue, anxiety and depression all decreased during treatment and that physical functioning increased, for both CBT and GET. Table 1 also shows that the effect size of both treatment modalities on the four outcome variables was large. Table 2 shows that treatment, regardless of modality, had a significant effect on fatigue, physical functioning, anxiety, and depression. In addition, it shows that when the two metacognitive change covariates (negative beliefs about thoughts concerning uncontrollability and danger, and cognitive confidence) were entered into the model, along with the anxiety and depression covariates, both (together with depression) had significant fixed effects on levels of fatigue. The fixed effect of modality of treatment was significant when anxiety and physical functioning were the outcome variables.

Table 3 shows the CGI and TSS outcomes at post-therapy and follow-up, as well as clinically useful change. These suggest that the majority of patients were satisfied with their treatment with roughly half who completed both treatment modalities reporting a positive change. Very few patients reported dissatisfaction with treatment or a negative change. Clinically useful change was assessed using the same method adopted by the PACE trial: i.e. an improvement of two or more in CFS scores and/or eight or more in SF-36 PF. Table 3 presents this data for both the entire sample and a subsample of patients who met PACE trial inclusion criteria (i.e. six or more on bimodal CFS and 60 or less on the SF-36 PF) to account for restriction of range issues.

\section{Discussion}

Together with the findings of the PACE trial (White et al., 2011), this evaluation provides further evidence that suggests that both CBT and GET are effective treatments for CFS. The analyses also suggest that both treatment options decrease fatigue, anxiety, and depression, as well as increase physical functioning. Both treatment options also appear to be satisfactory for the majority of patients and the overall completion rate was good, which may be attributed to the therapy assessment, affording the opportunity for patients to ask questions and make informed treatment choices, and therapists to identify potential obstacles to therapy (NICE, 2007). The CGI 
data is comparable to that obtained by the PACE trial (White et al., 2011) but the clinical change data is worse (for CBT, comparing PACE to this evaluation, it was 59\% versus 50\% and for GET $61 \%$ to $34.3 \%)$. This difference seems due to treatments in this study impacting more on fatigue than physical functioning. It is worth noting that the end point for follow-up measures was 12 months for the PACE trial and at or after session 14 for patients in this service, and this could represent as little as six months between pre-treatment and follow-up. It may be that both CBT and GET require sustained application of therapeutic strategies for their full benefit to be felt.

Treatment modality was driven by patient choice and this may explain differences in baseline levels of anxiety and physical functioning, and consequently the variation in outcomes between CBT and GET. Anxious patients may choose CBT over GET because they are more concerned by physical activity or because CBT seems a more 'natural fit' with how they prioritise their symptoms. Similarly, those with lower physical functioning may perceive GET as more appropriate for their needs. Differences in baseline levels of anxiety and physical functioning mirrors the difference in treatment effects between the two treatment modalities and maybe reflective of a 'restriction of range': i.e. patients with higher anxiety and lower physical functioning have more 'room to improve'.

Changes in depression, beliefs about thoughts concerning uncontrollability and danger, and cognitive confidence were found to produce significant effects in the regression equations when fatigue severity was the outcome variable, regardless of treatment modality. The relationship between changing levels of depression and severity of fatigue symptoms could be explained in several ways. For example, levels of depression may reduce as patients’ symptoms are ameliorated. Alternatively, CBT treatment protocols for depression and CFS may share common features, and it may be this overlap that, in part, explains changing levels of depression. Finally, GET uses exercise as a central therapeutic tool and this has been shown to reduce depression (Cooney et al., 2013).

The relationship between fatigue levels and metacognitions is more difficult to explain. While it is possible that metacognitions were indirectly addressed in CBT (Wells, 2011), this is 
unlikely to be the case in GET. Beliefs about thoughts concerning uncontrollability and danger might lead to negative appraisals of cognitive experiences, consequently activating perseverative cognitive processes such as worry and rumination. Such perseverative thinking may not only increase negative effect, leading to physiological changes that may alter the experience of fatigue and disrupt restorative sleeping patterns, but also require effort that may contribute to mental fatigue. Alternatively, it could be that the relationship between beliefs about thoughts concerning uncontrollability and danger and changes in fatigue severity might reflect reductions in worry and symptom preoccupation - variables that have been shown to mediate outcomes in GET (MossMorris et al., 2005).

Metacognitions about lack of cognitive confidence may reflect the cognitive difficulties reported by individuals with CFS. Increases in cognitive confidence may simply be a consequence of improved concentration and memory, resulting from reductions in fatigue. However, metacognitions about cognitive confidence may also result in the inhibition of adaptive coping strategies when fatigued. For example, individuals with low cognitive confidence may be inclined to worry about perceived poor cognitive performance, and this effortful cognitive process may contribute to the severity of mental fatigue. The failure to find a significant effect of the need to control thoughts in the developmental models, which would be a parallel finding to the study by Maher-Edwards et al. (2011), may be explain the choice of outcome variable. In the earlier study, the outcome variables used were the subscales of the CFQ (mental and physical) rather than the total fatigue score used in this study.

Earlier research has suggested other mediators of therapeutic change in CFS. For example, it seems that reductions in fear-avoidance beliefs and focus on fatigue mediate change in CBT (Stahl, Rimes, \& Chalder, 2014; Wiborg, Knoop, Prins, \& Bleijenberg, 2011) and in GET (Moss-Morris et al., 2005). Arguably, from an MCT perspective, symptom focus may form part of the CAS because it is an attentional strategy potentially fuelled by metacognitions (Fernie, Maher-Edwards, Murphy, Nikcevic, \& Spada, 2014). Recent research has suggested that change in fear-avoidance beliefs is 
more important in GET than CBT for predicting treatment outcome (Chalder, Goldsmith, White, Sharpe, \& Pickles, 2015), even though this is not transparently targeted in the former. While this implies that directly targeting a mediator (such as fear-avoidance or, possibly, metacognition) is not necessary for change, it does not indicate whether mediator-targeted treatment would increase the magnitude of change.

This study is subject to some limitations that are unique to routine clinical practice. Firstly, only patients who consented for their data to be used were included in this analysis, and if they did not provide consent at all three data points, their data was withdrawn. Secondly, patients not consenting for their data to be used in such study may be reflective of dissatisfaction with treatment modalities offered. Thirdly, more confounding variables exist in routine clinical practice when compared to RCTs. For example, in routine clinical practice a wider range of patients tend to be seen with physical and psychiatric comorbidities, less commonly seen in patients in a RCT. Also, there is less control over treatment fidelity in routine clinical practice when compared to RCTs. Fourthly; we did not have a sufficient sample size to adequately power an analysis that modelled all five MCQ-30 factors and/or interaction effects, limiting an interpretation of the relative contribution of specific metacognitive factors to therapeutic change in CFS. Fifthly, psychiatric comorbidity was not fully clinically assessed and is not included in the data analysis. However, this study presents baseline anxiety and depression scores for both CBT and GET patients, which provides some sense of patients' emotional state. Finally, condition characteristics such as symptom duration were not recorded in the dataset, preventing the analysis of their role.

In conclusion, the findings from the current study suggest that CFS treatment protocols for CBT and GET, based on those from the PACE trial, achieve similar-to-poorer outcomes in routine clinical practice as in a RCTs. Both CBT and GET met with high levels of patient satisfaction, suggesting that such treatments are well received despite the controversy with which they are sometimes associated. 


\section{References}

Aggarwal, V. R., McBeth, J., Zakrzewska, J. M., Lunt, M., \& Macfarlane, G. J. (2006). The epidemiology of chronic syndromes that are frequently unexplained: do they have common associated factors? Int $J$ Epidemiol, 35(2), 468-476. doi: 10.1093/ije/dyi265

Chalder, T., Berelowitz, G., Pawlikowska, T., Watts, L., Wessely, S., Wright, D., \& Wallace, E. P. (1993). Development of a fatigue scale. Journal of Psychosomatic Research, 37(2), 147-153.

Chalder, T., Goldsmith, K. A., White, P. D., Sharpe, M., \& Pickles, A. R. (2015). Rehabilitative therapies for chronic fatigue syndrome: a secondary mediation analysis of the PACE trial. The Lancet Psychiatry.

Cooney, G. M., Dwan, K., Greig, C. A., Lawlor, D. A., Rimer, J., Waugh, F. R., . . Mead, G. E. (2013). Exercise for depression. Cochrane Database Syst Rev, 9.

Deale, A., Chalder, T., \& Wessely, S. (1998). Illness beliefs and treatment outcome in chronic fatigue syndrome. J Psychosom Res, 45(1), 77-83.

Fernie, B. A., Maher-Edwards, L., Murphy, G., Nikcevic, A. V., \& Spada, M. M. (2014). The Metacognitions about Symptoms Control Scale: Development and Concurrent Validity. Clin Psychol Psychother, n/a-n/a. doi: 10.1002/cpp.1906

Guy, W. (1976). ECDEU assessment manual for psychopharmacology: US Department of Health, Education, and Welfare, Public Health Service, Alcohol, Drug Abuse, and Mental Health Administration, National Institute of Mental Health, Psychopharmacology Research Branch, Division of Extramural Research Programs.

Hou, R., Moss-Morris, R., Bradley, B. P., Peveler, R., \& Mogg, K. (2008). Attentional bias towards healththreat information in chronic fatigue syndrome. Journal of Psychosomatic Research, 65(1), 47-50.

IBM. (2011). Statistical Package for the Social Sciences Version 20: SPSS IBM Chicago^ eIL IL.

Knoop, H., Prins, J. B., Moss-Morris, R., \& Bleijenberg, G. (2010). The central role of cognitive processes in the perpetuation of chronic fatigue syndrome. Journal of Psychosomatic Research, 68(5), 489-494.

Maher-Edwards, L., Fernie, B. A., Murphy, G., Nikcevic, A. V., \& Spada, M. M. (2012). Metacognitive Factors in Chronic Fatigue Syndrome. Clin Psychol Psychother, 19(6), 552-557. doi: 10.1002/cpp.757 
Maher-Edwards, L., Fernie, B. A., Murphy, G., Wells, A., \& Spada, M. M. (2011). Metacognitions and negative emotions as predictors of symptom severity in chronic fatigue syndrome. $J$ Psychosom Res, 70(4), 311-317. doi: 10.1016/j.jpsychores.2010.09.016

Moss-Morris, R., \& Petrie, K. J. (2003). Experimental evidence for interpretive but not attention biases towards somatic information in patients with chronic fatigue syndrome. Br J Health Psychol, 8(Pt 2), 195-208. doi: 10.1348/135910703321649169

Moss-Morris, R., Sharon, C., Tobin, R., \& Baldi, J. C. (2005). A randomized controlled graded exercise trial for chronic fatigue syndrome: outcomes and mechanisms of change. J Health Psychol, 10(2), 245259.

Mykletun, A. (2001). Hospital Anxiety and Depression (HAD) scale: factor structure, item analyses and internal consistency in a large population. The British Journal of Psychiatry, 179(6), 540-544. doi: 10.1192/bjp.179.6.540

NICE. (2007). Diagnosis and Management of Chronic Fatigue Syndrome. CG53. London: National Institute for Health and Clinical Excellence (NICE).

Prins, J. B., Bleijenberg, G., Bazelmans, E., Elving, L. D., de Boo, T. M., Severens, J. L., . . Van der Meer, J. W. (2001). Cognitive behaviour therapy for chronic fatigue syndrome: a multicentre randomised controlled trial. The Lancet, 357(9259), 841-847.

Quarmby, L., Rimes, K. A., Deale, A., Wessely, S., \& Chalder, T. (2007). Cognitive-behaviour therapy for chronic fatigue syndrome: comparison of outcomes within and outside the confines of a randomised controlled trial. Behav Res Ther, 45(6), 1085-1094.

Sharpe, M. C., Archard, L. C., Banatvala, J. E., Borysiewicz, L. K., Clare, A. W., David, A., . . et al. (1991). A report--chronic fatigue syndrome: guidelines for research. J R Soc Med, 84(2), 118-121.

Spada, M. M., Mohiyeddini, C., \& Wells, A. (2008). Measuring metacognitions associated with emotional distress: Factor structure and predictive validity of the metacognitions questionnaire 30. Personality and Individual Differences, 45(3), 238-242. 
Stahl, D., Rimes, K. A., \& Chalder, T. (2014). Mechanisms of change underlying the efficacy of cognitive behaviour therapy for chronic fatigue syndrome in a specialist clinic: a mediation analysis. Psychol Med, 44(6), 1331-1344. doi: 10.1017/S0033291713002006

Ware, J. E., \& Kosinski, M. (2001). SF-36 physical \& mental health summary scales: a manual for users of version 1: Quality Metric.

Ware Jr, J. E., \& Sherbourne, C. D. (1992). The MOS 36-item short-form health survey (SF-36): I. Conceptual framework and item selection. Medical Care, 473-483.

Wells, A. (2011). Metacognitive therapy for anxiety and depression: Guilford press.

Wells, A., \& Cartwright-Hatton, S. (2004). A short form of the metacognitions questionnaire: properties of the MCQ-30. Behav Res Ther, 42(4), 385-396. doi: 10.1016/S0005-7967(03)00147-5

White, P., Goldsmith, K., Johnson, A., Potts, L., Walwyn, R., DeCesare, J., . . Cox, D. (2011). Comparison of adaptive pacing therapy, cognitive behaviour therapy, graded exercise therapy, and specialist medical care for chronic fatigue syndrome (PACE): a randomised trial. The Lancet, 377(9768), 823836.

White, P. D., Sharpe, M. C., Chalder, T., DeCesare, J. C., \& Walwyn, R. (2007). Protocol for the PACE trial: A randomised controlled trial of adaptive pacing, cognitive behaviour therapy, and graded exercise as supplements to standardised specialist medical care versus standardised specialist medical care alone for patients with the chronic fatigue syndrome/myalgic encephalomyelitis or encephalopathy. BMC Neurol, 7(1), 6.

Wiborg, J. F., Knoop, H., Prins, J. B., \& Bleijenberg, G. (2011). Does a decrease in avoidance behavior and focusing on fatigue mediate the effect of cognitive behavior therapy for chronic fatigue syndrome? Journal of Psychosomatic Research, 70(4), 306-310.

Zigmond, A. S., \& Snaith, R. P. (1983). The hospital anxiety and depression scale. Acta Psychiatr Scand, 67(6), 361-370. 
Table 1: Means, standard deviations, and effect sizes across treatment modalities for fatigue (CFQ), physical functioning (SF-36 PF), anxiety (HADS-A) and depression (HADS-D)

\begin{tabular}{|c|c|c|c|c|c|c|c|c|}
\hline & \multicolumn{2}{|c|}{ CFQ } & \multicolumn{2}{|c|}{ SF-36 PF } & \multicolumn{2}{|c|}{ HADS-A } & \multicolumn{2}{|c|}{ HADS-D } \\
\hline & $\begin{array}{l}\text { CBT } \\
\text { (SD) } \\
\end{array}$ & $\begin{array}{l}\text { GET } \\
\text { (SD) }\end{array}$ & $\begin{array}{l}\text { CBT } \\
\text { (SD) }\end{array}$ & $\begin{array}{l}\text { GET } \\
\text { (SD) }\end{array}$ & $\begin{array}{l}\text { CBT } \\
\text { (SD) } \\
\end{array}$ & $\begin{array}{l}\text { GET } \\
\text { (SD) }\end{array}$ & $\begin{array}{l}\text { CBT } \\
\text { (SD) }\end{array}$ & $\begin{array}{l}\text { GET } \\
\text { (SD) }\end{array}$ \\
\hline Pre-treatment & $\begin{array}{l}22.96 \\
(5.74)\end{array}$ & $\begin{array}{l}22.49 \\
(6.22)\end{array}$ & $\begin{array}{l}61.18 \\
(28.96)\end{array}$ & $\begin{array}{l}51 \\
(25.05)\end{array}$ & $\begin{array}{l}10.18 \\
(4.37)\end{array}$ & $\begin{array}{l}8.26 \\
(4.44)\end{array}$ & $\begin{array}{l}8.21 \\
(4.10)\end{array}$ & $\begin{array}{l}7.66 \\
(4.01)\end{array}$ \\
\hline Post-treatment & $\begin{array}{l}15.86 \\
(7.74)\end{array}$ & $\begin{array}{l}17.71 \\
(9.15)\end{array}$ & $\begin{array}{l}65.76 \\
(24.75)\end{array}$ & $\begin{array}{l}60.29 \\
(25.60)\end{array}$ & $\begin{array}{l}8.04 \\
(3.51)\end{array}$ & $\begin{array}{l}6.97 \\
(4.20)\end{array}$ & $\begin{array}{l}5.12 \\
(2.93)\end{array}$ & $\begin{array}{l}5.71 \\
(3.89)\end{array}$ \\
\hline Follow-up & $\begin{array}{l}14.71 \\
(7.85)\end{array}$ & $\begin{array}{l}15.46 \\
(7.70)\end{array}$ & $\begin{array}{l}68.96 \\
(25.30)\end{array}$ & $\begin{array}{l}61.00 \\
(26.70)\end{array}$ & $\begin{array}{l}7.64 \\
(3.08)\end{array}$ & $\begin{array}{l}6.47 \\
(4.67)\end{array}$ & $\begin{array}{l}5.08 \\
(3.63)\end{array}$ & $\begin{array}{l}4.83 \\
(3.75)\end{array}$ \\
\hline $\begin{array}{l}\text { Effect size } \\
\text { (Cohen's } d)\end{array}$ & .97 & .83 & .36 & .50 & .75 & .61 & .90 & .91 \\
\hline
\end{tabular}


Table 2: Estimates of fixed effects for four linear mixed model regression analyses

\begin{tabular}{|c|c|c|c|}
\hline & Estimation & $\mathbf{T}$ & $\boldsymbol{P}$ \\
\hline \multicolumn{4}{|l|}{ Outcome variable: CFQ (Fatigue) } \\
\hline 1. Intercept & 11.21 & 8.82 & $<.001$ \\
\hline 2. Treatment modality & -.49 & -.57 & .57 \\
\hline 3. $\quad$ Timepoint 1 & 5.46 & 7.82 & .001 \\
\hline 4. Timepoint 2 & .80 & 1.09 & .28 \\
\hline 5. $\quad$ Timepoint 3 & - & - & - \\
\hline 6. HADS-Anxiety & 0.18 & 1.68 & .09 \\
\hline 7. HADS-Depression & 0.71 & 6.90 & $<.001$ \\
\hline 8. MCQ-30-Negative Beliefs about Thoughts & -0.26 & -2.40 & .02 \\
\hline 9. MCQ-30-Cognitive Confidence & 0.17 & 1.68 & $<.05$ \\
\hline \multicolumn{4}{|l|}{ Outcome variable: SF-36 PF (Physical Functioning) } \\
\hline 1. $\quad$ Intercept & 57.39 & 16.59 & $<.001$ \\
\hline 2. Treatment modality & 8.17 & 2.02 & $<.05$ \\
\hline 3. $\quad$ Timepoint 1 & -8.98 & -5.39 & $<.001$ \\
\hline 4. Timepoint 2 & -2.18 & -1.42 & .16 \\
\hline 5. Timepoint 3 & - & - & - \\
\hline \multicolumn{4}{|l|}{ Outcome variable: HADS-Anxiety } \\
\hline 1. Intercept & 6.48 & 11.27 & $<.001$ \\
\hline 2. Treatment modality & 1.74 & 2.64 & $<.01$ \\
\hline 3. $\quad$ Timepoint 1 & 2.13 & 6.65 & $<.001$ \\
\hline 4. Timepoint 2 & .34 & 1.34 & .18 \\
\hline 5. $\quad$ Timepoint 3 & - & - & - \\
\hline \multicolumn{4}{|l|}{ Outcome variable: HADS-Depression } \\
\hline 6. Intercept & 5.60 & 10.50 & $<.001$ \\
\hline 7. Treatment modality & .01 & .01 & .99 \\
\hline 8. $\quad$ Timepoint 1 & 2.93 & 10.01 & $<.001$ \\
\hline 9. $\quad$ Timepoint 2 & .25 & 1.19 & .24 \\
\hline 10. Timepoint 3 & - & - & - \\
\hline
\end{tabular}


Table 3: Clinical Global Impression (CGI), Therapy Satisfaction Scale (TSS), and clinically useful change outcomes at post-treatment and follow-up

\begin{tabular}{|c|c|c|c|}
\hline & & CBT (\%) & GET (\%) \\
\hline & & \multicolumn{2}{|c|}{ CGI } \\
\hline \multirow{3}{*}{ Post-Treatment } & Positive change & 50.5 & 51.2 \\
\hline & Minimal change & 49.5 & 47.6 \\
\hline & Negative change & 0.0 & 1.2 \\
\hline \multirow{3}{*}{ Follow-up } & Positive change & 49.4 & 42.1 \\
\hline & Minimal change & 48.2 & 50.0 \\
\hline & Negative change & 2.4 & 7.9 \\
\hline & & \multicolumn{2}{|c|}{ TSI } \\
\hline \multirow{3}{*}{ Post-Treatment } & Satisfied & 97.7 & 95.4 \\
\hline & Mostly indifferent & 2.3 & 2.3 \\
\hline & Dissatisfied & 0.0 & 2.3 \\
\hline \multirow{3}{*}{ Follow-up } & Satisfied & 93 & 97.4 \\
\hline & Mostly indifferent & 5.9 & 2.6 \\
\hline & Dissatisfied & 1.1 & 0.0 \\
\hline & & \multicolumn{2}{|c|}{ Clinically useful change in fatigue (CFS) } \\
\hline \multirow[t]{3}{*}{ Post-treatment } & Entire sample $(\mathrm{n}=148)$ & 62.1 & 62.2 \\
\hline & PACE inclusion criteria $(n=80)$ & 58.3 & 65.6 \\
\hline & Entire sample & 62.1 & 60.0 \\
\hline \multirow[t]{2}{*}{ Follow-up } & PACE inclusion criteria & 60.4 & 56.3 \\
\hline & & \multicolumn{2}{|c|}{$\begin{array}{c}\text { Clinically useful change in physical } \\
\text { functioning (SF-36 PF) }\end{array}$} \\
\hline \multirow[t]{3}{*}{ Post-treatment } & Entire sample & 35.0 & 46.7 \\
\hline & PACE inclusion criteria & 47.9 & 50.0 \\
\hline & Entire sample & 37.9 & 42.2 \\
\hline \multirow[t]{2}{*}{ Follow-up } & PACE inclusion criteria & 56.3 & 43.8 \\
\hline & & \multicolumn{2}{|c|}{$\begin{array}{c}\text { Clinically useful change in fatigue and } \\
\text { physical functioning }\end{array}$} \\
\hline \multirow[t]{3}{*}{ Post-treatment } & Entire sample & 30.1 & 37.8 \\
\hline & PACE inclusion criteria & 41.7 & 43.8 \\
\hline & Entire sample & 33.0 & 33.3 \\
\hline Follow-up & PACE inclusion criteria & 50.0 & 34.4 \\
\hline
\end{tabular}

